\title{
History of Cometary Exploration at Kyiv University
}

\author{
K.I. Churyumov \\ Astronomical Observatory of Kyiv National University
}

\begin{abstract}
The comet explorers at Kyiv University took part in numerous international observational programs for study of comets: C/1973 E1 Kohoutek in 1974, 27P/Crommelin in 1984, $1 \mathrm{P} /$ Halley in 1986, 23P/Brorsen-Metcalf in 1989, D/Shoemaker-Levy 9 in 1994, C/1996 B2 Hyakutake in 1996, C/1995 O1 Hale-Bopp in 1997, C/1999 S4 (LINEAR) in 2000 and others. Two comets, 67P/Churyumov-Gerasimenko in 1969 and C/1986 N1 (Churyumov-Solodovnikov) in 1986, were discovered. During 1939-2000 more than 1000 scientific papers devoted to comets were published in different journals, books and proceedings.
\end{abstract}

Active cometary exploration at Kyiv University in Ukraine began in 1939 when the astronomy department of the physics faculty of this university was founded by Prof. Sergey Konstantinovich Vsekhsvyatsky, who moved for this aim from Moscow to Kyiv. Prof. Vsekhsvyatsky played a major role in the development of cometary astronomy at Kyiv University. Vsekhsvyatsky's monographs "Physical characteristics of comets" (Moscow, 1958; Jerusalem, 1964) and "Nature and origin of comets and meteor matter" (Moscow, 1967) are widely known. On the initiative of Vsekhsvyatsky, in the village of Lisnyky (near Kyiv) the Comet station of Kyiv University was organized; here two optical telescopes (AZT-8 with mirror by $D=70 \mathrm{~cm}$ and AZT-14 with mirror $D=50 \mathrm{~cm}$ ) for photographic, photoelectric, TV and CCD observations of comets - were installed. In 1965 the special cometary project for observation and detection of comets began. During the term of this project two comets, 67P/ChuryumovGerasimenko in 1969 and C/1986 N1 (Churyumov-Solodovnikov) in 1986, were discovered. The comet explorers at Kyiv University took part in numerous international observational programs for the study of comets: C/1973 E1 Kohoutek in 1973-1974, 27P/Crommelin in 1984, 1P/ Halley in 1986, 23P / Brorsen-Metcalf in 1989, D/Shoemaker-Levy 9 in 1994, C/1996 B2 Hyakutake in 1996, C/1995 O1 Hale-Bopp in 1997, C/1999 S4 (LINEAR) and others. Between 1963 and 1992, 428 issues of Comet Circulars were published in Kyiv University. From 1939 to 2000 over 400 specialists were trained in the Astronomy Department of Kyiv University, many of whom became well known investigators of comets (O. Dobrovol'sky, V. Konopleva, H. Nazarchuk, O. Demenko, L. Shul'man, S. Gerasimenko and others). During the years 1939-2000, more than 1000 scientific papers devoted to comets were published in a variety of journals, books and proceedings.

A new phase in the study of the physics and dynamics of comets started at the University in the 1980 s when the famous comet Halley entered the in- 
ner Solar System. The International Halley Watch (IHW) and the Soviet program SOPROCH invited astronomers to participate in these programs. Prof. S.K. Vsekhsvyatsky and Dr K.I. Churyumov were included on the Coordinated Committee of SOPROCH. In 1984-85 astronomers of Kyiv University (KU) K. Churyumov, V. Tarashchuk and V. Kleshchonok obtained much observational material about comet Halley on astrometric, spectroscopic, electrophotometric (with narrow band filters), polarimetric, large scale and near nucleus phenomena, and visual photometry. Unique large scale pictures of comet Halley at the prime focus of the 100-cm Zeiss reflector were obtained on Dec 12, 16 and 17 in 1985, and Jan 7-8 and May 11 in 1986; on these photographs, for the first time in world history, were detected ring structures in the comet's plasma tail and also a rare phenomenon of the axial disconnection of the plasma tail. Spectral observations of comet Halley were also carried out.

Unique spectra of comet Scoritchenko-George were obtained on Feb 26, 1990 by K.I. Churyumov together with observers from the SAO of the RAS with the spectral TV-scanner and the CCD-matrix spectrograph installed on the world's largest telescope (before the 1990s): the 6-m BTA reflector on Mount Pastukhov in the Northern Caucasus. Several spectra of comets Levy (in 1990), Tanaka-Machholz (1991), Swift-Tuttle (1992), Schaumasse (1992), Hyakytake (1996) and Hale-Bopp (1996-1997) were also obtained.

Further unique observational material obtained was the speed electrophotometry, spectra, TV and CCD-images of the jovian satellites and new spots in the jovian atmosphere during the time of the demise of "comet of the century" - short period comet Shoemaker-Levy 9. In 1992 this comet was broken up by tidal forces in the Roche region of Jupiter into 21-23 secondary nuclei. Two years later these fragments collided with Jupiter in jovian latitudes -40 to -45 degrees.

Selected references are cited below.

\section{References}

Churyumov, K.I., Rozenbush, V.K., Rspayev F.K., \& Gorodetsky, D.I. 1990, Some results of the narrowband photometry of comet P/Halley (1986 III), AJ, 356, 687

Churyumov, K.I. 1996, Life of astronomer S.K. Vsekhsvyatskij as seen by students and colleagues, The International Comet Quarterly, 18, No.4

Churyumov, K.I., Evtushevsky, O.M., \& Kravtsov F.I. 1999, Motion of the near-nucleus shells of comet Hale-Bopp and rotation of its nucleus, in "Evolution and source regions of asteroids and comets" (IAU Colloquium No.173), p265

Prokpof'eva, V.V., Tarashchuk, V.P., \& Churyumov K.I. 1997, On spectral peculiarities recorded by fast monitoring during the collision of comet SL-9 with Jupiter, Astron and Astrophys Trans, 13, 199 